A. Pramesh Rao, G. Swarup and Gopal-Krishna, eds.

\title{
BVRI-Photometry of Distant Radio Galaxies from RC Catalogue in SAO RAS
}

\author{
Yu.N. Parijskij \\ Special Astrophysical Observatory, Nizhnij Arkhyz, Russia, 369167 \\ W.M. Goss \\ NRAO, Socorro, New Mexico 87801, USA
A.I. Kopylov, N.S. Soboleva, O.V. Verkhodanov, A.V. Temirova and O.P.Zhelenkova
Special Astrophysical Observatory, Nizhnij Arkhyz, Russia, 369167

\begin{abstract}
With the aim to extend a sample of the distant radio objects, about 100 steep spectrum FRII radio galaxies (SS FRII RG) from the RATAN-600 RC catalog were mapped by the VLA ${ }^{1}$ and identified with optical objects down to $R=24^{m}-25^{m}$ with the $6 \mathrm{~m}$ telescope. The mean estimated redshift of SS FRII RGs from the RC list happened to be greater than 1.5. BVRI photometry was made, and by standard model fitting we have estimated "colour" redshift and the age of stellar population of the parent $\mathrm{gE}$ galaxy in 50 cases. Several objects were found in which active star formation began in the first billion of years after the Big Bang.
\end{abstract}

To get a maximum information about distant radio sources discovered in the experiment "Cold" (Parijskij et al., 1991, 1992) the "Big Trio" project was suggested (Goss et al. 1992). It unified the RATAN-600, VLA and the $6 \mathrm{~m}$ Russian optical telescope.

A very steep spectrum (VSS, $\alpha \geq 0.9$ ) sample of about 100 radio sources with $3.9 \mathrm{GHz}$ flux in the range of $10-300 \mathrm{mJy}$ was selected and observed (after VLA mapping) with the $6 \mathrm{~m}$ telescope using CCD with $R_{c}$ filter. About $60 \%$ of the objects have been observed with $B V R_{c} I_{c}$ filters. A standard data reduction was done (Kopylov et al., 1995) with the ESO-MIDAS. The full set of our CCD $R_{c}$ images, VLA maps as well as tables with radio and optical parameters of all objects observed are given in Parijskij et al. (1996). The median value of identified objects is $m_{R}=22^{\mathrm{m}} 5$. There are 33 compact steep spectrum (CSS) objects in the sample 16 of which has $1^{\prime \prime}<L A S<4^{\prime \prime}$ and 17 are unresolved or barely resolved $\left(L A S<1^{\prime \prime}\right)$. 65 objects look like FRII and about 20 of them belong presumably to the most distant generation of RGs. 16 objects were classified as quasars by their stellar appearance on CCD images.

\footnotetext{
${ }^{1}$ The Very Large Array of the National Radio Astronomy Observatory is a facility of the National Science Foundation operated under cooperative agreement by Associated Universities, Inc.
} 
As was shown by Verkhodanov et al. (1999) BVRI colours are sufficient for accurate estimation of $z$ and age in the redshift range of 0.5-3.5. This method was checked on a sample of 45 radio galaxies published in literature and carefully selected as steep spectrum high $z$ objects of the FRII type. However, this "calibrating" sample was not homogeneous.

We have implemented this approach for 50 FRII and CSS radio galaxies of the RC catalogue that had been observed in $B, V, R_{c}, I_{c}$ bands during 1994-1998 with CCD camera on $6 \mathrm{~m}$ telescope. Our sample of VSS radio galaxies is now the largest one with at least four-band optical photometry. The data obtained were used to estimate colour photometric redshifts $\left(z_{\text {phot }}\right)$ and ages of host galaxies by comparison with two models of evolution of spectral energy distribution (SED) (Fioc and Rocca-Volmerange, 1997; Poggianti, 1997).

As the best example of high redshift population of our sample we present $S_{3.9 \mathrm{GHz}}=33 \mathrm{mJy}$ radio galaxy RC J0105+0501 $\left(m_{V}=22^{m} .5\right)$ which shows the colour properties and the structure characteristic of very distant powerful radio galaxies. In the $V$ band, the galaxy is most extended and is by $1^{m} .5$ brighter than in the $B$ band, which is interpreted almost unambiguously for the given class of objects as a powerful Ly $\alpha$ line emission and continuum depression in the adjacent region of shorter wavelengths. The negative colour index $V-R_{c}=-0.3$ and the small index $R_{c}-I_{c}=0.4$ are in agreement with this interpretation of the data. The redshift is estimated to be $3.4 \pm 0.3$. In $V$-band the host galaxy is resolved into two components separated by $1^{\prime \prime} .7$. The SW-component is reliably detected on the $B$ frame and may be an active nucleus of a radio galaxy. The second component may then be either a region of star formation induced by the jet or a gaseous cloud ionized by the radiation from the active nucleus or a combination of both.

For a homogeneous representative sample of 50 radio galaxies selected by flux density, radio spectrum and FRII type the $B V R I$ photometrical data have been obtained. Using evolutionary model SEDs we have estimated that the mean multicolour $(B V R I)$ age of the stellar population of the parent galaxies is of order of $1 \mathrm{Gyr}$, and that at least in some objects active star formation had begun in the first Gyr after the Big Bang. Such distant objects must be of high density contrast and modern cosmology has to explain this very early appearance of dense and massive protogalaxies, with quickly formed massive black holes inside them to produce FRII structures. Several objects have a cosmic age greater than the age of the Universe for $z$ obtained in SCDM cosmology, but $\Lambda$-term may help to resolve this problem.

\section{References}

Fioc M. \& Rocca-Volmerange B. 1997, A\&A, 326, 950

Goss W.M. et al. 1992, AZh, 69, 673

Kopylov A.I. et al. 1995, AZh, 72, 613

Parijskij Y.N. et al. 1991, A\&AS, 87, 1; 1992, A\&AS, 96, 583

Parijskij Yu.N. et al., 1996, Bull.SAO, 40, 5

Poggianti B.M. 1997, A\&AS, 122, 399

Verkhodanov O.V. et al. 1999, Bull.SAO, 48, 41, astro-ph/9910559 